\title{
ÉPOCA de APLICACIÓn y TOXICIDAd VARIETAL DEL HERBICIDA amicarbazone en la Caña de Azúcar, en Veracruz, México ${ }^{1}$
}

\author{
Application Time and Varietal Toxicity of the Herbicide Amicarbazone in Sugarcane in Veracruz, \\ Mexico
}

\author{
ESQUEDA-ESQUIVEL, V.A. ${ }^{2}$ y ROSALES-ROBLES, E. ${ }^{3}$
}

\begin{abstract}
RESUMEN - Durante el ciclo primavera-verano 2005, se establecieron tres experimentos en Úrsulo Galván, Ver., México, con el objetivo de determinar la mejor época de aplicación del herbicida amicarbazone en la caña de azúcar en condiciones de riego e identificar la susceptibilidad de las tres principales variedades cultivadas en el estado a este herbicida. En un experimento, se evaluó el control de malezas con amicarbazone a 0,7, 1,05 y 1,4 $\mathrm{kg}^{\mathrm{ha}^{-1}}$ aplicado en cuatro épocas: preemergencia antes del riego de germinación, preemergencia después del riego de germinación, postemergencia temprana y postemergencia tardia. En los otros experimentos, se evaluó la toxicidad de amicarbazone a 0, 0,7, 1,4 y 2,1 $\mathrm{kg} \mathrm{ha}^{-1}$, aplicado en preemergencia y postemergencia en las variedades de caña de azúcar Mex 69-290, CP 72-2086 y Mex 79-431. E1 quelite rastrero (Amaranthus lividus) fue mejor controlado con aplicaciones postemergentes de amicarbazone, a partir de 0,7 $\mathrm{kg} \mathrm{ha}^{-1}$. Por su parte, el control del zacate Guinea (Megathyrsus maximus) fue bajo en todas las épocas de aplicación. En aplicaciones preemergentes, el amicarbazone hasta $2,1 \mathrm{~kg} \mathrm{ha}^{-1}$ fue altamente selectivo a todas las variedades evaluadas, mientras que, cuando fue aplicado en postemergencia, ocasionó ligera toxicidad a las tres variedades de caña de azúcar, la cual fue mayor conforme se incrementó la dosis. Sin embargo, los daños desaparecieron entre los 30 y 45 días después de la aplicación y no ocasionaron reducción permanente en la altura de las plantas.
\end{abstract}

Palabras-clave: control de malezas, preemergencia, postemergencia, Saccharum officinarum, Amaranthus lividus, Megathyrsus maximus.

\begin{abstract}
During the 2005 Spring-Summer growing cycle, three experiments were established in Ursulo Galvan, Ver., Mexico, to determine the best time of application of the herbicide amicarbazone in irrigated sugarcane, and the susceptibility of the three main varieties grown in this state to this herbicide. In one experiment, weed control of amicarbazone at $0.7,1.05$ and $1.4 \mathrm{~kg} \mathrm{ha}^{-1}$ applied at four stages (pre-emergence before germination irrigation, pre-emergence after germination irrigation, early post-emergence and late post-emergence) was evaluated. In the other experiments, the toxicity of amicarbazone at $0,0.7,1.4$ and $2.1 \mathrm{~kg} \mathrm{ha} \mathrm{h}^{-1}$ was evaluated when applied at pre-emergence and post-emergence on sugarcane varieties Mex 69-290, CP 72-2086 and 79 Mex-431. Amaranthus lividus was better controlled with post-emergence applications of amicarbazone, starting at $0.7 \mathrm{~kg} \mathrm{ha-1}$. Control of Guinea grass (Megathyrsus maximus) was low with amicarbazone at all stages. In preemergence applications, amicarbazone up to $2.1 \mathrm{~kg} \mathrm{ha}^{-1}$ was highly selective to all varieties evaluated, whereas when applied in post-emergence, it caused slight toxicity to the three sugarcane varieties, increasing as the dose increased. However, the damages disappeared between 30 and 45 days after application, without causing a permanent reduction in plant height.
\end{abstract}

Keywords: weed control, pre-emergence, post-emergence, Saccharum officinarum, Amaranthus lividus, Megathyrsus maximus.

Recebido para publicação em 19.6.2012 e aprovado em 26.12.2012.

2 Ph.D., Investigador del Programa de Sanidad Forestal y Agrícola del Campo Experimental Cotaxtla, INIFAP, Km 34 carretera Veracruz-Córdoba, CP 94277, Medellín de Bravo, Ver. México, <esqueda.valentin@inifap.gob.mx>3 Ph.D., Investigador del Programa de Sanidad Forestal y Agrícola del Campo Experimental Río Bravo, INIFAP, Km 61 carretera Matamoros-Reynosa, CP 88900, Cd. Río Bravo, Tam., México, <enrique_77840@yahoo.com>.

Planta Daninha, Viçosa-MG, v. 31, n. 3, p. 611-621, 2013 


\section{INTRODUCCIÓN}

Uno de los factores más críticos en el proceso productivo de la caña de azúcar es la competencia de malezas con el cultivo, lo que es particularmente importante durante los primeros tres meses a partir de la germinación, cuando el crecimiento del cultivo es lento y su follaje no logra cubrir las malezas (Ramón et al., 1989). Esta competencia puede reducir la cantidad y la calidad de la caña de azúcar cosechada, además de disminuir el número de cortes económicamente viables. En Cuba, Díaz (2004) encontró que la competencia de las malezas a lo largo de 30 días, redujo el rendimiento de la caña de azúcar en $43 \%$, mientras que, con la competencia permanente, prácticamente se perdió todo el cultivo. Otros autores han reportado reducciones de $61,8 \%$ (Chaila et al., 2004) y 56,9\% (Zafar et al., 2010) en el rendimiento de este cultivo a causa de la competencia con varias especies de malezas durante diferentes períodos de tiempo. Para evitar o reducir la interferencia de las malezas, es necesario controlarlas de modo eficiente, sobre todo durante el período de emergencia de las plantillas o rebrote de las socas, hasta que el follaje del cultivo inhiba la nacencia o desarrollo de las plantas competidoras.

En México, la aplicación de herbicidas selectivos es el principal método empleado para el control de malezas en caña de azúcar (Esqueda et al., 2001). Los más utilizados son la ametrina y el diurón (Esqueda, 1999), que en algunas ocasiones son inconsistentes en el control de malezas (Esqueda, 1999) y pueden ocasionar toxicidad a algunas variedades (Osgood et al., 1972; Rodrigues et al., 2010).

La efectividad en el control de malezas y su selectividad con relación a la caña de azúcar pueden ser afectadas por la humedad del suelo en el momento de la aplicación la dosis, la variedad (Esqueda et al., 2001) y la época de aplicación (Monquero et al., 2009a). Algunos cultivares presentan respuestas diferenciadas a los herbicidas y tienen, como consecuencia, frecuentes problemas de fitotoxicidad, 1o que llega a ocasionar la reducción de la productividad de los cultivares más sensibles (Araldi, 2010).
Monquero et al. (2009b) encontraron diferencias significativas en el control de Euphorbia heterophylla e Ipomoea grandiflora en caña de azúcar, al aplicar diferentes herbicidas en preemergencia y en postemergencia. Por otra parte, muchos de los herbicidas preemergentes utilizados en la caña de azúcar son selectivos debido a que el suelo actúa como una zona de aislamiento que evita el contacto de las raíces y las yemas de la caña de azúcar con concentraciones altas de herbicidas (Richard Jr. \& Dalley, 2006). La toxicidad de la mezcla de oxifluorfén + ametrina en la caña de azúcar fue significativamente mayor cuando se aplicó en postemergencia que en preemergencia, aunque, en ambos casos, los daños se redujeron cuando las plantas crecieron. Por su parte, Azania et al. (2006) indicaron que los herbicidas metribuzina e isoxaflutole y las mezclas de diurón + hexazinona y de azafenidin + hexazinona ocasionaron mayor toxicidad cuando fueron aplicados en postemergencia tardía que cuando la aplicación se realizó en postemergencia temprana.

E1 amicarbazone [4-amino-N-(1,1dimetiletil)-4,5-dihidro-3-(1-metiletil)-5-oxo1H-1,2,4-triazole-1-carboxamida] es un herbicida del grupo químico de las triazolinonas cuyo modo de acción es la inhibición de la fotosintesis en las plantas susceptibles (Dayan et al., 2009), lo que ocasiona reducción de crecimiento, clorosis y necrosis de los tejidos foliares, que puede provocar la muerte de las plantas. Controla tanto malezas de hoja ancha como gramíneas, aunque su efecto puede variar en diferentes especies (Negrisoli et al., 2007; Carbonari et al., 2009). Amicarbazone es absorbido tanto por las raíces como por el follaje, por lo que puede aplicarse tanto en preemergencia como en postemergencia a las malezas presentes en la caña de azúcar (Francino et al., 2010).

En México, no existe información sobre el comportamiento del amicarbazone en caña de azúcar. Por eso, teniendo en cuenta la hipótesis de que hay diferencia en el control de malezas cuando se aplica amicarbazone en diferentes dosis y épocas, y que este herbicida es selectivo a las principales variedades de caña de azúcar que se siembran en México, se establecieron tres experimentos con dos 
objetivos: el de identificar la efectividad de tres dosis de amicarbazone en el control de malezas, cuando se aplica el herbicida en la caña de azúcar y en las malezas en preemergencia, antes y después del riego de germinación, y en postemergencia temprana y tardía; y también el de determinar la selectividad de cuatro dosis de amicarbazone en tres variedades de caña de azúcar, cuando fueron aplicadas en fases de preemergencia y postemergencia, en las condiciones agroecológicas en que se produce este cultivo en el estado de Veracruz, principal productor de caña de azúcar en el país.

\section{MATERIAL Y MÉTODOS}

Los tres experimentos se establecieron en Monte de Oro, municipio de Úrsulo Galván, Ver., México, dentro del área de abasto del Ingenio La Gloria S. A. El predio tiene un suelo franco arcilloso con $\mathrm{pH}$ de 7,2 y un contenido de materia orgánica de 1,4\%. El clima de la región es del tipo $\mathrm{Aw}_{1}$, que corresponde a los cálidos subhúmedos, con una precipitación media anual de $1.188 \mathrm{~mm}$, una humedad relativa con promedio anual de $81,3 \%$ y una temperatura media de $24,8{ }^{\circ} \mathrm{C}$. Las siembras de los experimentos se realizaron entre el 13 y el 15 de abril de 2005. En el experimento para determinar la época de aplicación (experimento 1), se utilizó caña de azúcar de la variedad Mex 69-290, en una densidad de $12.000 \mathrm{~kg} \mathrm{ha}^{-1}$. A su vez, en los experimentos para determinar la toxicidad a la caña en preemergencia (experimento 2) y postemergencia (experimento 3), se utilizaron las variedades Mex 69-290, CP 72-2086 y Mex 79-431, que son las más utilizadas en México, en la misma densidad de siembra.

\section{Experimento 1}

Se evaluaron 12 tratamientos, resultantes de la combinación de tres dosis de amicarbazone: 0,$7 ; 1,05 ; \mathrm{y} 1,4 \mathrm{~kg}$ de ingrediente activo ha ${ }^{-1}$ con cuatro épocas de aplicación: Preemergencia antes del riego de germinación (PREAR), Preemergencia después del riego de germinación (PREDR), Postemergencia temprana (POSTM) y Postemergencia tardía (POSTD).
Se utilizó el diseño experimental de bloques al azar con cuatro repeticiones y arreglo de tratamientos en parcelas divididas. Las parcelas grandes fueron las épocas de aplicación de amicarbazone, y las pequeñas, las dosis del herbicida. Las parcelas pequeñas estaban compuestas de dos surcos de $6 \mathrm{~m}$ de largo y $1,30 \mathrm{~m}$ de separación, en los que se aplicaron los tratamientos correspondientes, más un surco sin aplicación compartido con la parcela vecina, de tal manera que cada parcela de dos surcos con aplicación estaba bordeada por surcos sin aplicación, utilizados como testigos enhierbados laterales, para auxiliar en la evaluación visual del control de malezas.

Los tratamientos se aplicaron con un aspersor motorizado de mochila, equipado con un aguilón y cuatro boquillas de abanico plano 8003. Los tratamientos 1, 2 y 3 se aplicaron el 14 de abril de 2005, en la fase de preemergencia, en la caña de azúcar y en las malezas y con terreno seco. Los tratamientos 4,5 y 6 se aplicaron el 20 de abril de 2005, en la preemergencia, en la caña de azúcar y en las malezas, tres días después del riego de germinación. Los tratamientos 7,8 y 9 se aplicaron el 9 de mayo de 2005, cuando la caña de azúcar tenía de tres a cinco hojas, la altura de los zacates variaba entre 8 y $20 \mathrm{~cm}$, y la de las malezas de hoja ancha, entre 4 y $13 \mathrm{~cm}$. Los tratamientos 10,11 y 12 se aplicaron el 20 de mayo de 2005, cuando la caña de azúcar tenía entre cinco y seis hojas, la altura de los zacates variaba entre 20 y $40 \mathrm{~cm}$, y la de las malezas de hoja ancha, entre 10 y $30 \mathrm{~cm}$. En las aplicaciones realizadas en postemergencia, realizadas el 9 y 20 de mayo, se añadió un surfactante no iónico (Agridex) a $0,25 \% \mathrm{v} / \mathrm{v}$.

Un día después de la aplicación de los tratamientos 1,2 y 3 , se aplicó el riego de germinación y se realizaron cuatro riegos de auxilio a cada 10 a 15 días. No fue necesario el uso de insecticidas ni de fungicidas durante el tiempo que se condujo el experimento.

Para determinar la densidad de población de malezas, se utilizó un cuadro de 0,5 x 0,5 $\mathrm{m}$, lanzado al azar en dos ocasiones en cada una de las parcelas correspondientes a los tratamientos POSTM y POSTD, inmediatamente antes de su aplicación. Las malezas 
contenidas en el interior del cuadro fueron identificadas por especie y cuantificadas, y se produjeron las transformaciones necesarias para determinar su densidad de población por hectárea.

E1 control de las especies de malezas dominantes por los diferentes tratamientos se determinó de forma visual mediante la escala de 0 a $100 \%$, en la que 0 significó que las malezas no fueron afectadas, y 100, que fueron completamente destruidas. Las evaluaciones se realizaron a los 15, 45 y 90 días después de la aplicación (DDA). Debe indicarse que, en la primera evaluación de los tratamientos de 1 a 6 , no pudo asignarse un porcentaje de control, pues había muy poca emergencia de malezas, tanto en los surcos tratados como en los de los testigos enhierbados laterales. Se evaluó visualmente la toxicidad de los tratamientos herbicidas en la caña de azúcar a los 15, 30 y 45 DDA. Para esto, se utilizó la escala de 0 a $100 \%$, en que 0 significó que la caña no fue afectada, y $100 \%$, que fue completamente destruida.

Se efectuaron análisis de varianza de los datos experimentales, y como prueba de comparación de medias se utilizó Tukey $(p>0,05)$.

\section{Experimentos 2 y 3}

En cada experimento, se evaluaron 12 tratamientos, que resultaron de la combinación de cuatro dosis de amicarbazone: $0 ; 0,7 ; 1,4 ; \mathrm{y}$ $2,1 \mathrm{~kg}$ de ingrediente activo ha ${ }^{-1}$ con tres variedades de caña de azúcar: Mex 69-290, CP 72-2086 y Mex 79-431.

En ambos experimentos, se utilizó el diseño experimental de bloques al azar con cuatro repeticiones y arreglo de tratamientos en parcelas divididas. Las parcelas grandes estaban compuestas por las variedades de caña de azúcar, y las pequeñas, por las dosis de amicarbazone. Las parcelas experimentales pequeñas estaban constituidas por dos surcos de $8 \mathrm{~m}$ de longitud, espaciados 1,30 m entre sí.

En el experimento 2, los tratamientos se aplicaron, en preemergencia, en la caña de azúcar y en las malezas el 20 de abril de 2005, tres dias después del riego de germinación.
La aplicación se realizó con el aspersor indicado anteriormente.

Por su parte, en el experimento 3, los tratamientos se aplicaron el 9 de mayo de 2005, cuando la variedad Mex 69-290 tenía de cuatro a seis hojas y altura entre 42 y $57 \mathrm{~cm}$; la variedad CP 72-2086, de cuatro a cinco hojas y altura de entre 35 y $60 \mathrm{~cm}$; y la variedad Mex 79-431, de tres a cinco hojas y altura de entre 26 y $49 \mathrm{~cm}$. La aplicación se realizó de la manera indicada con anterioridad. Se añadió un surfactante no iónico (Agridex) a $0,25 \% \mathrm{v} / \mathrm{v}$.

En ambos experimentos, las parcelas no aplicadas fueron utilizadas como testigos absolutos. En los dos experimentos, se evaluó visualmente la toxicidad de las dosis de amicarbazone en las tres variedades de caña de azúcar, a los 15, 30, 45 y 60 DDA, utilizando la escala de 0 a $100 \%$. También se midió la altura de cinco plantas de caña de azúcar de cada parcela experimental a los 30,45 y 60 DDA, y se realizó un análisis de varianza de los datos. Como prueba de comparación de medias, se utilizó Tukey $(\mathrm{p}>0,05)$.

\section{RESULTADOS Y DISCUSIÓN}

\section{Experimento 1}

Densidad de población de malezas. En el momento de la aplicación de los tratamientos POSTM (9 de mayo), la población total de malezas era de 768.000 plantas por hectárea, mientras que, cuando se aplicaron los tratamientos POSTD (20 de mayo), la población era de 448.000 plantas por hectárea. El zacate Guinea (Megathyrsus maximus) de la familia Poaceae y el quelite rastrero (Amaranthus lividus) de la familia Amaranthaceae fueron las especies dominantes, que ocupaban juntos el $86,46 \%$ y $73,21 \%$ de la población total de malezas el 9 y 20 de mayo, respectivamente. Otras dos especies de malezas de la familia Euphorbiaceae: el gusanillo (Acalypha sp.) y la pata de paloma (Croton lobatus), fueron encontradas en poblaciones bajas, y su control por todos los tratamientos fue semejante al observado para el quelite rastrero, por lo que no se incluyen sus datos en este escrito.

Control de Megathyrsus maximus. A los 15 DDA, los tratamientos POSTM mostraron 
promedios de controles de $M$. maximus cercanos a los $55 \%$, más que el doble de los que se obtuvieron con las aplicaciones POSTD. Con respecto a las dosis de amicarbazone, se observó una tendencia a incrementar el control de esta especie, a medida que se aumentaban las dosis aplicadas, aunque los valores se pueden considerar muy bajos (Cuadro 1).

A los 45 DDA, los tratamientos PREDR tenían un control de $M$. maximus superior a $50 \%$, estadísticamente semejante al obtenido con los tratamientos POSTM. Esto indica que, para el control de esta especie con aplicaciones preemergentes de amicarbazone, la humedad del suelo es importante en el momento de aplicar el herbicida, como indicaron Negrisoli et al. (2007) para Brachiaria decumbens, y que las plantas pequeñas son más afectadas que aquellas más desarrolladas. A su vez, los tratamientos aplicados en PREAR y POSTD demostraron controles de esta especie inferiores a $20 \%$. Al comparar el efecto de las dosis de amicarbazone, se determinó que, si se aplica 1,05 o 1,4 $\mathrm{kg} \mathrm{ha}^{-1}$, se obtiene un control de $M$. maximus estadísticamente superior al que se obtiene con la aplicación de $0,7 \mathrm{~kg} \mathrm{ha}^{-1}$ (Cuadro 1).

A los 90 DDA, con las aplicaciones PREDR el control promedio de $M$. maximus era solamente ligeramente superior a $30 \%$, mientras que, con los tratamientos aplicados en las otras épocas, el control fue menor a $15 \%$. Con respecto al comportamiento de las dosis de amicarbazone, se conservó la superioridad de
1,05 y $1,4 \mathrm{~kg} \mathrm{ha}^{-1}$ con relación a $0,7 \mathrm{~kg} \mathrm{ha}^{-1}$ (Cuadro 1).

Los resultados obtenidos en este trabajo indican que $M$. maximus es una maleza de dificil control con amicarbazone en las diferentes épocas de aplicación, por lo que no se debe utilizar este herbicida como un tratamiento individual cuando esta especie sea dominante. Una posible explicación para el bajo control es el reducido efecto del amicarbazone en la actividad fotosintética de $M$. maximus, como indicaron Gomes et al. (2010), lo que puede estar relacionado con la velocidad de absorción de este herbicida, que, de acuerdo con Velini et al. (2010), varia en las diferentes especies de malezas. Sin embargo, otros autores han verificado controles eficientes de esta maleza con amicarbazone (Carbonari, 2007; Pizzo et al., 2010). Tal situación de bajo control también se observa con otras especies de malezas gramineas anuales, por ejemplo, Azania et al. (2010) encontraron un control deficiente de $B$. decumbens con amicarbazone, mientras que Negrisoli et al. (2007) indicaron que el control de esta especie varía de acuerdo con la presencia o ausencia de paja de caña de azúcar y si la aplicación de una lluvia simulada ocurre antes o después de la aplicación del amicarbazone.

Por 1o anterior, se debe recomendar amicarbazone para el control de una maleza específica en pre o postemergencia con base en los resultados de estudios locales y no en aquellos obtenidos en regiones o países diferentes.

Cuadro 1 - Efecto de los factores época de aplicación y dosis de amicarbazone en el control del zacate Guinea (Megathyrsus maximus) (\%) en los cultivos de caña de azúcar de la variedad Mex 69-290 a los 15, 45 y 90 días de la aplicación (DDA)

\begin{tabular}{|c|c|c|c|c|c|c|c|c|c|c|c|c|}
\hline \multirow{3}{*}{$\begin{array}{l}\text { Época de } \\
\text { aplicación }\end{array}$} & \multicolumn{12}{|c|}{ Control de Megathyrsus maximus (\%) } \\
\hline & \multicolumn{4}{|c|}{$15 \mathrm{DDA}$} & \multicolumn{4}{|c|}{$45 \mathrm{DDA}$} & \multicolumn{4}{|c|}{90 DDA } \\
\hline & 0,7 & 1,05 & 1,4 & Promedio & 0,7 & 1,05 & 1,4 & Promedio & 0,7 & 1,05 & 1,4 & Promedio \\
\hline PREAR & $\bar{x}$ & $\bar{x}$ & $\bar{x}$ & $\bar{x}$ & 0,00 & 22,50 & 27,50 & $16,67 \mathrm{~b}$ & 0,00 & 5,00 & 12,50 & $5,83 \mathrm{bc}$ \\
\hline PREDR & $\mathrm{x}$ & $\mathrm{x}$ & $\mathrm{x}$ & $\mathrm{x}$ & 50,00 & 52,50 & 55,00 & $52,50 \mathrm{a}$ & 22,50 & 35,00 & 37,50 & $31,67 \mathrm{a}$ \\
\hline POSTM & 42,50 & 57,50 & 62,50 & 54,17 & 20,00 & 37,50 & 40,00 & $32,50 \mathrm{a}$ & 5,00 & 12,50 & 17,50 & $11,67 \mathrm{~b}$ \\
\hline POSTD & 12,50 & 23,80 & 32,50 & 22,92 & 0,00 & 13,80 & 22,50 & $12,08 \mathrm{~b}$ & 0,00 & 2,50 & 7,50 & $3,33 \mathrm{c}$ \\
\hline Promedio & 27,50 & 40,63 & 47,50 & & $17,50 \mathrm{~b}$ & $31,56 \mathrm{a}$ & $36,25 \mathrm{a}$ & & $6,88 \mathrm{~b}$ & $13,75 \mathrm{a}$ & $18,75 \mathrm{a}$ & \\
\hline
\end{tabular}

Valores medios de las últimas fila y columna seguidos de la misma letra no son diferentes de acuerdo a la prueba de Tukey $(p \leq 0,05)$. $\mathrm{x}=$ no fue evaluado por haber escasa emergencia de las malezas. PREAR $=$ Preemergencia antes del riego. PREDR $=$ Preemergencia después del riego. POSTM $=$ Postemergencia temprana. POSTD $=$ Postemergencia tardía. A los 15 DDA, se presentan sólo los promedios de los tratamientos en POSTM y POSTD 
Control de Amaranthus lividus. Se observó significancia estadística en épocas de aplicación, pero no en dosis de amicarbazone a los 45 y 90 DDA. La correlación épocas de aplicación $\mathrm{x}$ dosis de amicarbazone no se mostró significativa en ninguna de las evaluaciones.

A los 15 DDA, todos los tratamientos aplicados en postemergencia temprana y tardía demostraron un control total de $A$. lividus (Cuadro 2).

Tanto a los 45 como a los 90 DDA, se observó un control total de A. lividus con las dosis aplicadas en POSTD y casi total con las que se aplicaron en POSTM. En estas épocas de evaluación, las aplicaciones realizadas en postemergencia fueron significativamente superiores a las aplicadas en PREAR y PREDR, cuyos controles variaron entre 75 y $85 \%$ a los 45 DDA y 70 y $75 \%$ a los 90 DDA. Por otra parte, a partir de los 45 DDA, no se observó diferencia estadística en el control de A. lividus proporcionado por la aplicación de 0,7, 1,05 y $1,4 \mathrm{~kg} \mathrm{ha}^{-1}$ (Cuadro 2). Lo anterior difiere de lo encontrado para $M$. maximus, lo que indica que algunas especies son más sensibles al amicarbazone que otras.

En concordancia con lo anterior, Esqueda (2005) observó un mejor control de A. lividus y A. hybridus cuando el amicarbazone se aplicó en dosis de $0,7 \mathrm{~kg} \mathrm{ha}^{-1}$ o menores en postemergencia que cuando eso se realizó en preemergencia. Aunque el control de A. lividus sea claramente superior en postemergencia que en preemergencia, Leptochloa mucronata fue controlada de forma semejante en ambas épocas de aplicación con la dosis $1,25 \mathrm{~kg} \mathrm{ha}^{-1}$, pero, en dosis menores, las aplicaciones en postemergencia fueron significativamente superiores (Esqueda, 2005). Por eso, es importante determinar la época más adecuada de aplicación para controlar las diferentes especies dominantes de malezas en las plantaciones de caña de azúcar.

Toxicidad en la caña de azúcar. Se observó significancia estadística tanto en el factor época de aplicación como en la dosis de amicarbazone a los 15 y 30 DDA. No hubo significancia para las interacciones en ninguna de las épocas de evaluación.

Las plantas de caña de azúcar no presentaron daños cuando el amicarbazone se aplicó en PREAR o PREDR en cualquiera de las dosis evaluadas. Sin embargo, las plantas en que la aplicación se realizó en postemergencia mostraron ligera clorosis y presencia de áreas necróticas en las puntas, bordes y láminas foliares del tercio superior de algunas hojas. A los 15 DDA, los daños fueron significativamente mayores con las aplicaciones tardias $(11,4 \%)$ que con las tempranas $(4,1 \%)$. Por otra parte, al comparar la toxicidad ocasionada por las dosis de amicarbazone, se determinó que los menores daños se produjeron con la dosis de $0,7 \mathrm{~kg} \mathrm{ha}^{-1}$ y se incrementaron significativamente con la dosis de $1,05 \mathrm{~kg} \mathrm{ha}^{-1}$, así como con la de $1,4 \mathrm{~kg} \mathrm{ha}^{-1}$, que fue la que ocasionó la mayor toxicidad. A los 30 DDA, se redujo la toxicidad en las plantas en las que se realizó la aplicación en POSTD. Por ello, en

Cuadro 2 - Efecto de los factores época de aplicación y dosis de amicarbazone en el control del quelite rastrero (Amaranthus lividus) (\%) en los cultivos de caña de azúcar de la variedad Mex 69-290 a los 15, 45 y 90 días de la aplicación (DDA)

\begin{tabular}{|c|c|c|c|c|c|c|c|c|c|c|c|c|}
\hline \multirow{3}{*}{$\begin{array}{l}\text { Época de } \\
\text { aplicación }\end{array}$} & \multicolumn{12}{|c|}{ Control de Amaranthus lividus (\%) } \\
\hline & \multicolumn{4}{|c|}{15 DDA } & \multicolumn{4}{|c|}{$45 \mathrm{DDA}$} & \multicolumn{4}{|c|}{$90 \mathrm{DDA}$} \\
\hline & 0,7 & 1,05 & 1,4 & Promedio & 0,7 & 1,05 & 1,4 & Promedio & 0,7 & 1,05 & 1,4 & Promedio \\
\hline PREAR & $\mathrm{x}$ & $\mathrm{x}$ & $\mathrm{x}$ & $\mathrm{x}$ & 70,0 & 77,5 & 78,8 & $75,42 \mathrm{~b}$ & 67,5 & 75,0 & 81,3 & $74,58 \mathrm{~b}$ \\
\hline PREDR & $\mathrm{x}$ & $\mathrm{x}$ & $\mathrm{x}$ & $\mathrm{x}$ & 80,0 & 83,8 & 85,0 & $82,92 \mathrm{~b}$ & 72,5 & 73,8 & 76,3 & $74,17 \mathrm{~b}$ \\
\hline POSTM & 100,0 & 100,0 & 100,0 & 100,0 & 99,8 & 100,0 & 100,0 & $99,92 \mathrm{a}$ & 97,5 & 98,8 & 100,0 & $98,75 \mathrm{a}$ \\
\hline POSTD & 100,0 & 100,0 & 100,0 & 100,0 & 100,0 & 100,0 & 100,0 & $100,00 \mathrm{a}$ & 100,0 & 100,0 & 100,0 & $100,00 \mathrm{a}$ \\
\hline Promedio & 100,0 & 100,0 & 100,0 & & $87,44 \mathrm{a}$ & $90,31 \mathrm{a}$ & $90,94 \mathrm{a}$ & & $84,38 \mathrm{a}$ & $86,88 \mathrm{a}$ & $89,38 \mathrm{a}$ & \\
\hline
\end{tabular}

Valores medios de las últimas fila y columna seguidos de la misma letra no son diferentes de acuerdo a la prueba de Tukey $(p>0,05)$. $\mathrm{x}=$ no fue evaluado por haber escasa emergencia de las malezas. PREAR $=$ Preemergencia antes del riego. PREDR $=$ Preemergencia después del riego. POSTM $=$ Postemergencia temprana. POSTD $=$ Postemergencia tardía. A los 15 DDA, se presentan sólo los promedios de los tratamientos en POSTM y POSTD 
esta época, los daños ocasionados por los dos tipos de aplicaciones postemergentes eran estadisticamente semejantes entre sí e inferiores a $4 \%$. Con respecto a las dosis de amicarbazone, los daños fueron estadísticamente semejantes con 1,05 y $1,4 \mathrm{~kg} \mathrm{ha}^{-1} \mathrm{y}$ superiores a los ocasionados por $0,7 \mathrm{~kg} \mathrm{ha}^{-1}$ (Cuadro 3).

A los 45 DDA, la toxicidad ocasionada por los tratamientos postemergentes había desaparecido completamente (datos no mostrados).

Aunque en este trabajo los tratamientos preemergentes no hayan ocasionado toxicidad a la variedad Mex 69-290, en Brasil, con aplicaciones preemergentes de 0,875 a $1,4 \mathrm{~kg}$ de amicarbazone por hectárea, Francino et al. (2010) encontraron toxicidades menores que $10 \%$ en la variedad CTC 2, aunque estos valores son considerados bajos. Por su parte, al aplicar 1,4 kg de amicarbazone por hectárea en postemergencia, Pizzo et al. (2010) reportaron toxicidad entre 15 y $20 \%$ en el cultivar IACSP 93-3026 a los 15 DDA, daños que desaparecieron completamente a los 30 DDA, situación muy similar a lo encontrado en este trabajo con la misma dosis en postemergencia tardía.

\section{Experimento 2}

Toxicidad a la caña de azúcar en aplicación preemergente. Las variedades Mex 69-290 y Mex 79-431 no presentaron sintomas visuales de toxicidad producidos por la aplicación de amicarbazone en preemergencia en ninguna de las épocas de evaluación (datos no mostrados), lo que coincide con lo indicado por Esqueda (2005). A su vez, en las plantas de la variedad CP 72-2086, se verificó que el ángulo de contacto de las hojas con el tallo era ligeramente mayor ("hojas abiertas") en las plantas de caña de azúcar en las que se aplicó tal herbicida en dosis de 1,4 y 2,1 $\mathrm{kg} \mathrm{ha}^{-1}$, pero que no había clorosis, necrosis o reducción de vigor, por lo que no se le asignó un valor porcentual. Este efecto sólo se observó a los 15 y 30 DDA.

Altura de las plantas de caña de azúcar. Se encontró significancia estadística para el factor variedades en la altura de las plantas de caña de azúcar a los 30, 45 y 60 DDA, pero en ninguna de las épocas de evaluación hubo diferencias significativas entre diversas dosis de amicarbazone, ni la relación variedades $\mathrm{x}$ dosis de amicarbazone fue significativa.

Al promediar la altura de planta de cada una de las variedades de caña de azúcar tratadas con las diferentes dosis de amicarbazone, se observó que, a los 30 DDA, las plantas de la variedad Mex 69-290 estaban significativamente más altas que las de las variedades CP 72-2086 y Mex 79-431, cuyas alturas fueron estadísticamente semejantes entre sí. A su vez, en esta época, no hubo reducción significativa en la altura de planta debido a la aplicación de amicarbazone (Cuadro 4).

Tanto a los 45 como a los 60 DDA, la altura media de planta de las variedades Mex 69-290

Cuadro 3 - Efecto de los factores época de aplicación y dosis de amicarbazone en la toxicidad (\%) de la caña de azúcar de la variedad Mex 69-290 a los 15 y 30 DDA

\begin{tabular}{|c|c|c|c|c|c|c|c|c|}
\hline \multirow{3}{*}{$\begin{array}{l}\text { Época de } \\
\text { aplicación }\end{array}$} & \multicolumn{4}{|c|}{$15 \mathrm{DDA}$} & \multicolumn{4}{|c|}{$30 \mathrm{DDA}$} \\
\hline & \multicolumn{3}{|c|}{$\begin{array}{l}\text { Dosis de amicarbazone } \\
\qquad\left(\mathrm{kg} \mathrm{ha}^{-1}\right)\end{array}$} & \multirow{2}{*}{ Promedio } & \multicolumn{3}{|c|}{ Dosis de amicarbazone $\left(\mathrm{kg} \mathrm{ha}^{-1}\right)$} & \multirow{2}{*}{ Promedic } \\
\hline & 0,7 & 1,05 & 1,4 & & 0,7 & 1,05 & 1,4 & \\
\hline PREAR & 0,00 & 0,00 & 0,00 & $0,00 \mathrm{c}$ & 0,00 & 0,00 & 0,00 & $0,00 \mathrm{~b}$ \\
\hline PREDR & 0,00 & 0,00 & 0,00 & $0,00 \mathrm{c}$ & 0,00 & 0,00 & 0,00 & $0,00 \mathrm{~b}$ \\
\hline POSTM & 2,75 & 4,25 & 5,25 & $4,08 \mathrm{~b}$ & 2,25 & 3,50 & 4,50 & $3,42 \mathrm{a}$ \\
\hline POSTD & 8,75 & 10,50 & 15,00 & $11,42 \mathrm{a}$ & 2,25 & 3,00 & 3,75 & $3,00 \mathrm{a}$ \\
\hline Promedio & $2,88 \mathrm{c}$ & $3,69 \mathrm{~b}$ & $5,06 \mathrm{a}$ & & $1,13 \mathrm{~b}$ & $1,63 \mathrm{a}$ & $2,06 \mathrm{a}$ & \\
\hline
\end{tabular}

Valores medios de las últimas fila y columna seguidos de la misma letra no son diferentes de acuerdo a la prueba de Tukey $((p \leq 0,05)$ PREAR $=$ Preemergencia antes del riego. PREDR $=$ Preemergencia después del riego. POSTM $=$ Postemergencia temprana. POSTD $=$ Postemergencia tardía. 
y Mex 79-431 era estadísticamente semejante, y las plantas de esta última variedad tenían una altura semejante a la de la variedad CP 72-2086, que presentó las plantas más bajas. En ambas épocas de evaluación, aunque las plantas tratadas con la dosis mayor de amicarbazone fueron las más altas, no eran significativamente diferentes de las plantas tratadas con las dosis media y baja de este herbicida ni de las plantas sin aplicación (Cuadro 4).

De lo que se observó de la información obtenida en este experimento, se puede afirmar que la variación de la altura media entre las variedades se debe a diferencias genéticas entre ellas. Por otra parte, aunque con las dosis de 1,4 y $2,1 \mathrm{~kg}$ de amicarbazone en la variedad CP 72-2086 se hayan observado las hojas más "abiertas", este efecto fue temporal y no afectó la altura de planta. Por lo anterior, es posible concluir que el herbicida amicarbazone puede ser aplicado en preemergencia, sin generar riesgo de toxicidad para las variedades Mex 69-290, CP 72-2086 y Mex 79-431.

\section{Experimento 3}

Toxicidad a la caña de azúcar en aplicación postemergente. Se observó significancia estadística en el factor dosis de amicarbazone a $\operatorname{los} 15$ y 30 DDA. No hubo significancia para variedades de caña de azúcar, ni para la interacción entre ambos factores en ninguna de las épocas de evaluación.

Los daños ocasionados por el herbicida amicarbazone a las plantas de caña de azúcar consistieron en ligera clorosis y presencia de áreas necróticas en la punta, bordes y lámina foliar del tercio superior de algunas hojas, sintomas propios de los herbicidas inhibidores de la fotosíntesis. Además de los sintomas indicados anteriormente, en la variedad CP 72-2086, se observaron plantas con "hojas abiertas", tal como ocurrió con las aplicaciones preemergentes.

A los 15 DDA, las tres variedades de caña de azúcar mostraron síntomas de toxicidad debido a la aplicación del herbicida amicarbazone. Aunque la variedad Mex 69-290 haya mostrado los daños más altos, éstos no fueron estadísticamente diferentes de los ocasionados a las otras dos variedades (Cuadro 5).
En todas las variedades, la toxicidad se incrementó conforme aumentó la dosis del herbicida, y el daño ocasionado por las dosis aplicadas fue estadísticamente diferente entre ellas.

A los 30 DDA, se observó una reducción en la toxicidad en comparación con la registrada a los 15 DDA. No hubo diferencia significativa con relación a la toxicidad en las tres variedades de caña de azúcar y se mantuvo la diferencia estadística entre los daños ocasionados por las diferentes dosis de amicarbazone (Cuadro 5). A partir de los 45 DDA, no se detectaron sintomas de toxicidad en ninguna de las variedades. Aunque estos valores de toxicidad sean relativamente bajos y semejantes a los encontrados en diferentes variedades por otros autores (Esqueda, 2006; Correia \& Zeitoum, 2010), no se debe recomendar el uso de amicarbazone en variedades en que no se haya realizado antes una prueba de selectividad, pues han sido detectados cultivares con daños de hasta $70 \%$ en el follaje con aplicación de dosis comerciales de este herbicida (Soares, 2011).

Altura de las plantas de caña de azúcar. Se observó significancia estadística para el factor variedades de caña de azúcar a los 30, 45 y 60 DDA, con dosis de amicarbazone.

A los 30 y 45 DDA, las variedades Mex 69290 y Mex 79-431 tenian plantas con alturas estadísticamente semejantes entre sí y superiores a la de CP 72-2086. A su vez, ninguna dosis de amicarbazone ocasionó la reducción de la altura de las variedades de caña de azúcar en comparación con el testigo absoluto (Cuadro 6).

En la evaluación efectuada a los 60 DDA, las plantas de la variedad Mex 69-290 presentaron altura estadísticamente semejante a la de la variedad Mex 79-431 y superior a la de CP 72-2086, que, a su vez, tenía una altura semejante a la de la última variedad indicada. Con relación a las dosis de amicarbazone, la altura de las plantas tratadas con $1,4 \mathrm{~kg} \mathrm{ha}^{-1}$ fue significativamente menor que la de las plantas en que se aplicó $0,7 \mathrm{~kg}$ ha ${ }^{1}$. Sin embargo, fue estadísticamente similar a la de las plantas en que se aplicó $2,1 \mathrm{~kg} \mathrm{ha}^{-1} \mathrm{y}$ a la del testigo absoluto (Cuadro 6). 

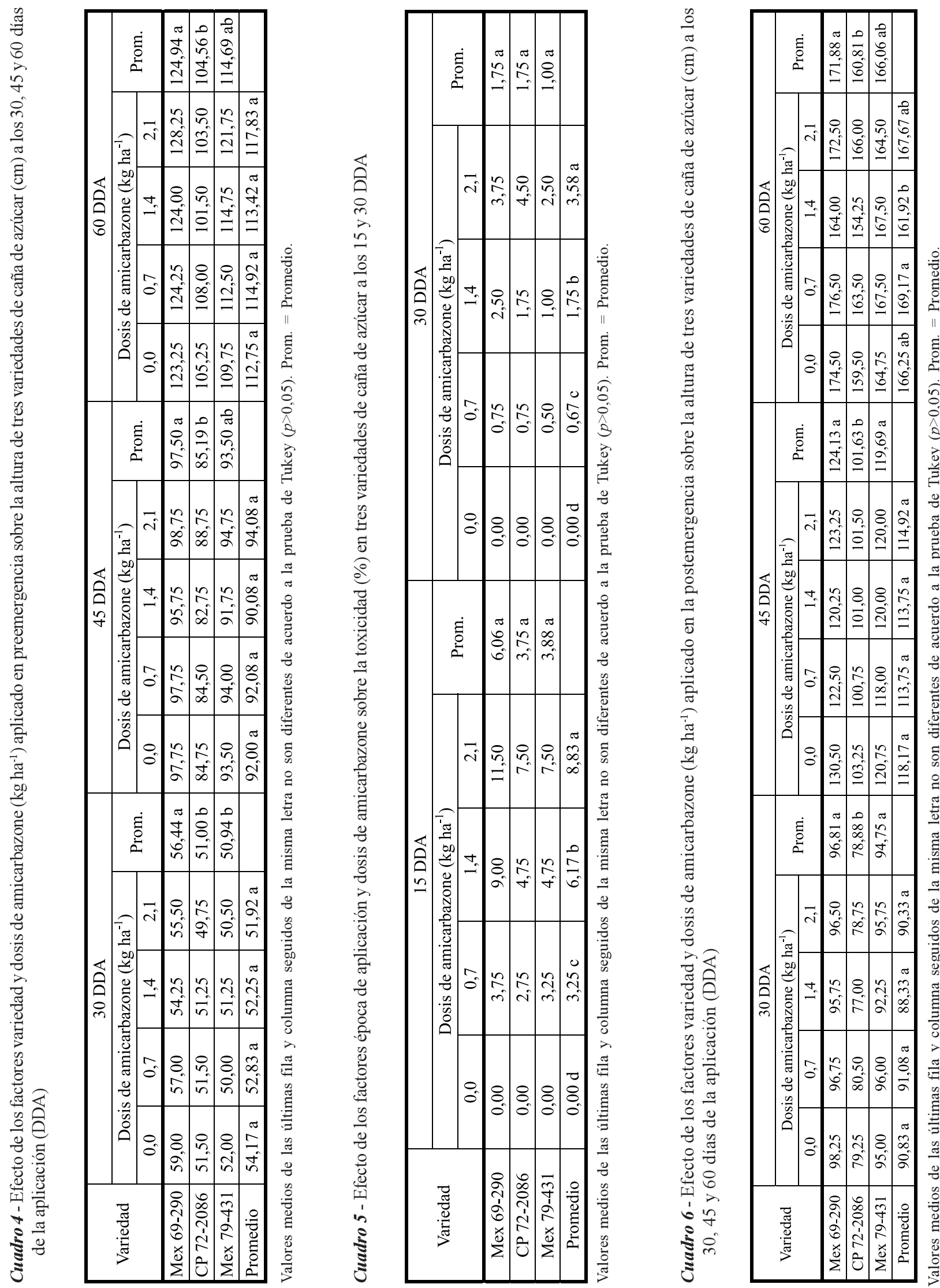
Teniendo en cuenta este experimento, se puede indicar que la toxicidad ocasionada por el herbicida amicarbazone aplicado en postemergencia aumenta conforme se incrementa la dosis del herbicida, pero en una dosis de $2,1 \mathrm{~kg} \mathrm{ha}^{-1}$, es semejante o menor a la de otros herbicidas que se recomiendan para el cultivo de caña de azúcar (Esqueda, 1999; Esqueda et al., 2001). La toxicidad desaparece entre los 30 y 45 DDA y no tiene efecto permanente sobre la altura de las tres variedades de caña de azúcar.

En conclusión, en condiciones de riego, el control de A. lividus es significativamente mayor con aplicaciones postemergentes de amicarbazone que con aplicaciones preemergentes, y es suficiente aplicar la dosis de 0,7 $\mathrm{kg} \mathrm{ha}^{-1}$. El control de $M$. maximus con amicarbazone es deficiente con las dosis y épocas de aplicación evaluadas. Amicarbazone aplicado en preemergencia hasta $2,1 \mathrm{~kg} \mathrm{ha}^{-1}$ es altamente selectivo a las variedades de caña de azúcar Mex 69-290 y Mex 79-431, y ocasiona temporalmente el efecto de "hojas abiertas" en la variedad CP 72-2086, pero no afecta la altura de planta de ninguna variedad. Amicarbazone aplicado en postemergencia, produce ligera toxicidad en las variedades de caña de azúcar Mex 69-290, Mex 79-431 y CP 72-2086. Tal efecto es mayor a medida que se incrementa la dosis aplicada. La toxicidad ocasionada por amicarbazone en postemergencia desaparece entre los 30 y 45 DDA y no ocasiona reducción permanente en la altura de planta de la caña de azúcar.

\section{AGRADECIMIENTOS}

Los autores agradecen a Arysta LifeScience México por el financiamiento de los experimentos que dieron origen a esta publicación.

\section{LITERATURA CITADA}

ARALDI, R. Avaliação da absorção do amicarbazone e intoxicação em cana-de-açúcar e plantas daninhas. 2010. 83 f. Dissertação (Mestrado em Agronomia/Proteção de Plantas) - Universidade Estadual Paulista "Júlio de Mesquita Filho", Botucatu, 2010

Planta Daninha, Viçosa-MG, v. 31, n. 3, p. 611-621, 2013
AZANIA, C. A. M. et al. Seletividade de herbicidas. III Aplicação de herbicidas em pós-emergência inicial e tardia da cana-de-açúcar na época da estiagem. Planta Daninha, v. 24, n. 3, p. 489-495, 2006.

AZANIA, C. A. M. et al. Evaluation of herbicides applied on sugar cane during rainy season in Brazil. R. Bras. Herb., v. 9, n. 1, p. 9-16, 2010.

CARBONARI, C. A. Eficácia do herbicida amicarbazone em aplicação conjunta com a colheita de cana-de-açúcar no controle das principais plantas daninhas da cultura. 2007. 119 f. Dissertação (Mestrado em Agronomia/Proteção de Plantas) - Universidade Estadual Paulista "Júlio de Mesquita Filho", Botucatu, 2007.

CARBONARI, C. A. et al. Efeitos de diferentes condições de umidade do solo e profundidades de germinação de Brachiaria plantaginea e Digitaria spp. sobre a eficácia do herbicida amicarbazone. R. Bras. Herbic., v. 8, n. 3 , p. $68-74,2009$

CHAILA, S. et al. Competencia de Sicyos polyacanthus en caña de azúcar. Planta Daninha, v. 22, n. 4, p. 545-551, 2004.

CORREIA, N. M.; ZEITOUM, V. Controle químico de melão-de-são-caetano em área de cana-soca. Bragantia, v. 69, n. 2 , p. $329-337,2010$.

DAYAN, F. E.; TRINDADE, M. L. B.; VELINI, E. D Amicarbazone, a new photosystem II inhibitor. Weed Sci., v. 57, n. 6, p. $579-583,2009$

DÍAZ, J. C. Influencia de la población y la distancia entre surcos sobre la competencia de las malezas en caña de azúcar. R. Protec. Veg., v. 19, n. 3, p. 177-178, 2004.

ESQUEDA, E. V. A. Control de malezas en caña de azúcar con clomazone y ametrina. Agron. Mesoamericana, v. 10, n. 2, p. 23-30, 1999

ESQUEDA, E.V.A. Control de malezas en caña de azúcar con amicarbazone. In: CONGRESO NACIONAL DE LA CIENCIA DE LA MALEZA, 26., 2005, Cd. Victoria, Tam., México. Memorias... Cd. Victoria, Tam.: 2005. p. 64-69.

ESQUEDA, E. V. A. Control de malezas con amicarbazone, solo y en mezcla con otros herbicidas en caña de azúcar de temporal. In: CONGRESO NACIONAL DE LA CIENCIA DE LA MALEZA, 27., 2006, Ensenada, B.C., México.

Memorias... Ensenada, B.C.:2006. p. 80-86.

ESQUEDA, V. A. et al. Evaluación de la mezcla ametrina + clomazone en caña de azúcar. Agron. Mesoamericana, v. 12, n. 2, p. 161-167, 2001. 
FRANCINO, P. et al. Seletividade do herbicida amicarbazone para soqueiras de cana-de-açúcar aplicado na época chuvosa no estado de Goiás. In: CONGRESSO BRASILEIRO DA CIÊNCIA DAS PLANTAS DANINHAS, 27., 2010, Ribeirão Preto. Palestras... Ribeirão Preto: 2010. p. 2327-2330.

GOMES, G. L. G. C. et al. Efeito de herbicidas na atividade fotossintética de plantas daninhas ocorrentes na cultura do milho. In: CONGRESSO BRASILEIRO DA CIÊNCIA DAS PLANTAS DANINHAS, 27., 2010, Ribeirão Preto.

Palestras... Ribeirão Preto: 2010. p. 3020-3024.

MONQUERO, P. A. et al. Eficácia de herbicidas aplicados em diferentes épocas sobre B. pilosa e I. quamoclit em área de cana-de-açúcar colhida mecanicamente. Planta Daninha, v. 27, n. 3 , p. $563-570,2009 a$

MONQUERO, P. A. et al. Eficácia de herbicidas aplicados em diferentes épocas e espécies daninhas em área de cana-deaçúcar colhida mecanicamente. Planta Daninha, v. 27, n. 3, p. $309-317,2009$ b.

NEGRISOLI, E. et al. Controle de plantas daninhas pelo amicarbazone aplicado na presença de palha de cana-deaçúcar. Planta Daninha, v. 25, n. 3, p. 603-611, 2007.

OSGOOD, R. V.; ROMANOWSKI, R. R.; HILTON, H. W. Differential tolerance of Hawaiian sugarcane cultivars to diuron. Weed Sci., v. 20, n. 6, p. 537-539, 1972.
PIZZO, I. V. et al. Seletividade e eficácia de controle de plantas daninhas pela associação entre óleo fúsel e herbicidas em cana-de-açúcar. Planta Daninha, v. 28, n. 2, p. 347-357, 2010 .

RAMÓN, M.; NASS, H.; MENDOZA, C. Efectividad de siete tratamientos químicos para el combate de malezas en caña de azúcar. Caña Azúcar, v. 7, n. 1, p. 17-32, 1989.

RICHARD Jr., E. P.; DALLEY, C. D. Sugarcane response to depth of soil cover at planting and herbicide treatment. J. Am. Soc. Sugar Cane Technol., v. 26, n. 1, p. 14-25, 2006.

RODRIGUES, F. R. et al. Tolerância diferencial de variedades de cana-de-açúcar a estresse por herbicidas. Bragantia, v. 69, n. 2, p. 395-404, 2010.

SOARES, R. O. et al. Herbicidas de diferentes mecanismos de ação e a seletividade a cultivares de cana-de-açúcar. Nucleus, v. 8, n. 1, p. 337-350, 2011.

VELINI, E. D. et al. Dinâmica de absorção foliar e ação de atrazina e amicarbazone. In: CONGRESSO BRASILEIRO DA CIÊNCIA DAS PLANTAS DANINHAS, 27., 2010, Ribeirão Preto. Palestras... Ribeirão Preto: 2010. p. 2980-2983.

ZAFAR, M. et al. Weed-crop competition effects on growth and yield of sugarcane planted using two methods. Pakistan J. Bot., v. 42, n. 2, p. 815-823, 2010. 